\title{
Short-term visual result after simultaneous photorefractive keratectomy and small-aperture cornea inlay implantation
}

This article was published in the following Dove Press journal:

Clinical Ophthalmology

II November 2016

Number of times this article has been viewed

\author{
Majid Moshirfar ${ }^{1,2}$ \\ Ryan T Wallace ${ }^{3}$ \\ David F Skanchy 4 \\ Jordan D Desautels ${ }^{5}$ \\ Steven H Linn' \\ Phillip C Hoopes Jr ${ }^{1}$ \\ Daniel S Durrie ${ }^{1,6}$
}

'Hoopes, Durrie, Rivera, Research Center, Hoopes Vision, Draper, UT,

${ }^{2}$ Department of Ophthalmology and Visual Sciences, John A. Moran Eye Center, University of Utah School of Medicine, Salt Lake City, UT,

${ }^{3}$ Brigham Young University, Provo, UT, ${ }^{4}$ McGovern Medical School, The University of Texas Health Science Center at Houston, Houston, TX, ${ }^{5}$ Tufts University School of Medicine, Boston, MA, ${ }^{6}$ Durrie Vision, Overland Park, KS, USA
Correspondence: Majid Moshirfar Hoopes, Durrie, Rivera, Research Center, Hoopes Vision, II820 South State Street, Suite \#200, Draper, UT 84020 , USA

$\mathrm{Tel}+\mathrm{I} 80 \mathrm{I} 5680200$

$\mathrm{Fax}+\mathrm{I} 8015630200$

Email cornea2020@me.com
Purpose: To report the short-term results of simultaneous photorefractive keratectomy (PRK) and small-aperture cornea inlay implantation (KAMRA) surgery in treating presbyopia.

Methods: Simultaneous PRK and KAMRA inlay surgery was performed on 21 patients from July 2015 to March 2016. Follow-up exams were conducted at 1, 3, and 6 months postoperatively. Our patients were also divided preoperatively into three categories: myopic, hyperopic, and emmetropic. Over the 6-month period, the main outcome measures were uncorrected near visual acuity (UNVA), changes in best-corrected distance visual acuity, and mean spherical equivalent refraction.

Results: At 6-month follow-up, 83\% (10/12) of patients had a monocular UNVA of 20/40 or better, and $75 \%$ had a binocular UNVA of 20/40 (J5) or better. At 6 months, the overall mean refractive spherical equivalent (MRSE) was $-0.60 \mathrm{D}( \pm 0.42$ standard deviation [SD], range: -1.38 to -0.13$)$ with a mean change of $-0.43 \mathrm{D}( \pm 1.19 \mathrm{SD}$, range: -1.5 to 2.63$)$ compared to preoperative data. Overall, $91 \%(10 / 11)$ of patients were within a $\pm 0.5 \mathrm{D}$ range of our target $-0.75 \mathrm{D}$ for KAMRA use. One-hundred percent (5/5) of the hyperopes, 50\% (1/2) of emmetropes, and 100\% (4/4) of myopes met the targeted range. At 6 months, the MRSE for the hyperopic subgroup $(n=5)$ was $0.33 \mathrm{D}( \pm 0.20 \mathrm{SD})$, the MRSE for the emmetropic subgroup $(\mathrm{n}=2)$ was $-1.19 \mathrm{D}( \pm 0.19 \mathrm{SD})$, and the MRSE for the myopic subgroup $(n=4)$ was $-0.66 \mathrm{D}( \pm 0.36 \mathrm{SD})$.

Conclusion: Based on preliminary results and a small sample size, it seems that simultaneous PRK and KAMRA is effective and predictable. There are multiple advantages in performing simultaneous PRK and KAMRA including a shorter recovery time and less steroid use than the two surgeries performed separately.

Keywords: KAMRA, PRKamra, PRK, femptosecond, corneal, presbyopia

\section{Introduction}

It is estimated that over one billion people worldwide have impaired near vision caused by presbyopia. ${ }^{1-3}$ The small-aperture inlay (KAMRA; AcuFocus, Inc, Irving, TX, USA) has shown effective results in the treatment of presbyopia, ${ }^{4-7}$ and in April 2015, the US Food and Drug Administration (FDA) approved its use in the US. Using the pinhole technique, the inlay focuses light as it enters the eye to improve the depth of field and reading vision. However, the KAMRA inlay is only approved for patients with between -0.75 and $+0.5 \mathrm{D}$ with $<0.75 \mathrm{D}$ of cylinder, excluding many hyperopic and myopic patients from receiving the procedure and its potential benefits. The aim of this study was to use the KAMRA inlay with photorefractive keratectomy (PRK) surgery simultaneously (PRKamra) to correct refractive error in patients for both near and distance vision in a different method than traditional monovision laser-assisted in situ keratomileusis (LASIK). 
To our knowledge, this is the first research of simultaneous PRK and KAMRA inlay surgeries and is the first information regarding the ongoing results in 21 eyes.

\section{Patients and methods}

\section{Patient characteristics}

Patient demographics are shown in Table 1. The median age of our patients was 51 years with a range of 46-63 years. Of our patient group, 62\% (13/21) were male, and $62 \%$ $(13 / 21)$ of the procedures were performed in the left eye. Monocular (mono UNVA) and binocular uncorrected near visual acuity (Bin UNVA) were 20/50 (J6) or worse for $86 \%(18 / 21)$ of patients with an average of logMAR of $0.5(20 / 63)( \pm 0.2$ standard deviation [SD], range: $0.2-0.8)$ and $0.5(20 / 63)( \pm 0.2 \mathrm{SD}$, range: $0.1-0.8)$, respectively. Preoperatively, the overall mean spherical equivalent refraction was $0.1 \mathrm{D}( \pm 1.0 \mathrm{SD}$, range: -3.5 to 1.4$)$ among 21 patients. Our patients were also divided preoperatively into three categories: myopic, hyperopic, and emmetropic. Of our patients, 52\% (11/21) were hyperopic preoperatively, and the MRSE for this hyperopic group was $0.68 \mathrm{D}( \pm 0.4 \mathrm{SD}$, range: $0.25-1.4)$. Thirty-three percent $(7 / 21)$ of our patients were myopic, and the MRSE for this group was $-0.77 \mathrm{D}( \pm 1.2 \mathrm{SD}$, range: -3.5 to -0.13$)$. In addition, $14 \%(3 / 21)$ of patients were emmetropic preoperatively, with an MRSE of 0.

This study was approved by the Hoopes, Durrie, Rivera Research Review Board. Before providing written informed consent, all patients were given detailed information regarding each treatment.

\section{Surgical technique}

The KAMRA inlay surgery was performed by marking the first purkinje reflex on the cornea using a $4 \mathrm{~mm}$ optical

Table I Patient demographics

\begin{tabular}{|c|c|}
\hline Parameter $(n=2 I)$ & Value \\
\hline Male sex, n (\%) & $61.9(13)$ \\
\hline Left eye OS, n (\%) & $61.9(13)$ \\
\hline Age median (range) & $51(46-63)$ \\
\hline Pocket depth mean (range) & $249(230-280)$ \\
\hline Mean monocular UNVA & $0.5(20 / 63) \pm 0.2$ SD $(0.2-0.8)$ \\
\hline \multicolumn{2}{|l|}{ logMAR/Snellen (range) } \\
\hline Mean binocular UNVA & $0.5(20 / 63) \pm 0.2$ SD $(0.1-0.8)$ \\
\hline \multicolumn{2}{|l|}{ logMAR/Snellen (range) } \\
\hline Mean spherical equivalent & $0.1 \pm I .0$ SD (-3.5 to I.4) \\
\hline \multicolumn{2}{|l|}{ of all groups } \\
\hline Hyperopic $(n=I I)$ & $0.7 \pm 0.4$ SD $(0.5-1.4)$ \\
\hline Emmetropic $(n=3)$ & 0 \\
\hline Myopic $(n=7)$ & $-0.8 \pm 1.2$ SD $(-3.5$ to -0.3$)$ \\
\hline
\end{tabular}

Abbreviations: OS, oculus sinister; UNVA, uncorrected near visual acuity; $\mathrm{SD}$, standard deviation. zone marker in the nondominant eye of the patient. If the distance between the purkinje and the center of the pupil was $<300 \mu \mathrm{m}$ apart, the purkinje image was used as the center. If the two were $>300 \mu \mathrm{m}$ apart, the centration mark was placed halfway between the center of the pupil and the purkinje reflex. Using a spot/line separation $\leq 6 \times 6 \mu \mathrm{m}$, a $150 \mathrm{kHz}$ IntraLase iFS femtosecond laser (Abbott Medical Optics, Inc, Santa Ana, CA, USA) was used to create a stromal corneal pocket between 220 and $280 \mu \mathrm{m}$ deep with a channel width of $4.7 \mathrm{~mm}$, offset $1.1 \mathrm{~mm}$. Subsequently, the KAMRA inlay was inserted using special loading forceps and centered with respect to the original marking.

Attention was then given to the PRK portion of the procedure. The corneal epithelium was scored using an $8.0 \mathrm{~mm}$ trephine, and using an $8.5 \mathrm{~mm}$ alcohol well, the cornea was exposed to a $20 \%$ alcohol solution for 30 seconds. Subsequently, the epithelium was removed. Laser ablation at a $6.5 \mathrm{~mm}$ zone with a $9 \mathrm{~mm}$ blend was performed using an Allegretto EX-500 excimer laser (Alcon, Fort Worth, TX, USA) and programmed to correct the patients' refractive error with a target refraction of $-0.75 \mathrm{D}$.

Patients subsequently received a bandage contact lens with fluoroquinolone and prednisolone acetate 1\% drops four times daily for the first week. Subsequently, the bandage contact lens and antibiotic drops were discontinued, and the prednisolone acetate $1 \%$ was continued four times a day for the first month. Afterward, the patient's steroid was changed to fluorometholone $0.1 \%$ which was tapered over the course of an additional 8 weeks. Postoperative follow-up for the simultaneous surgeries was performed at months 1,3 , and 6 .

\section{Study parameters}

Over the 6-month period, the study parameters were Mono UNVA, Bin UNVA, lines lost of monocular best-corrected distance visual acuity (Mono CDVA), and mean refractive spherical equivalent (MRSE).

\section{Results}

The preoperative and postoperative Mono UNVA and Bin UNVA are shown in Figures 1 and 2, respectively. Preoperatively, all 21 patients had a 20/20 or better Mono CDVA, and $76 \%(16 / 21)$ of patients had a Mono UDVA of 20/25 or better in the eye that was subsequently operated on. Preoperatively, $86 \%(18 / 21)$ of our patients had a Bin UNVA or Mono UNVA of 20/50 (J6) or worse.

At 1-month follow-up, 81\% (17/21) of our patients had a Mono UNVA of 20/40 or better in their nondominant eye. At 3-month follow-up, 92\% (11/12) of patients had a Mono UNVA of 20/40 (J5) or better, with 75\% (9/12) 


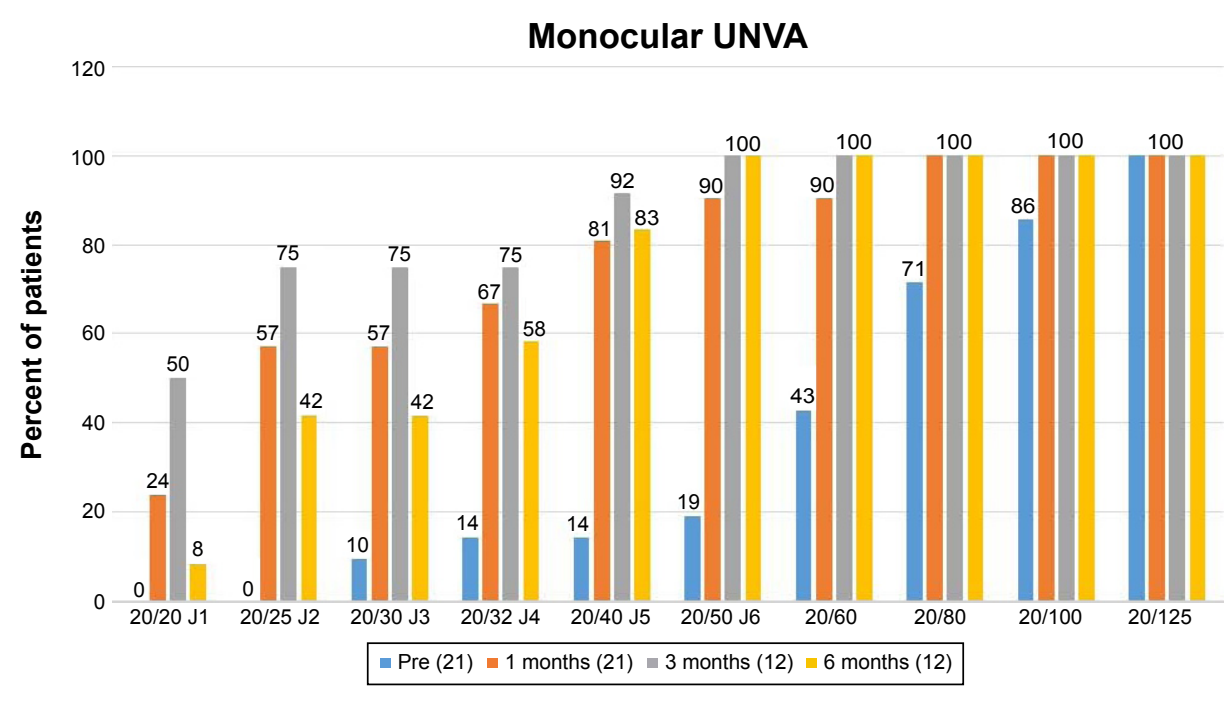

Figure I Cumulative monocular UNVA shown before KAMRA inlay implantation and at I, 3, and 6 months postoperatively. Abbreviation: UNVA, uncorrected near visual acuity.

seeing 20/25 (J2) or better. At 6-month follow-up, there was a slight drop as $83 \%(10 / 12)$ had a Mono UNVA of at least 20/40 (J5), with 42\% (5/12) seeing 20/25 (J2) or better. At 1 month, 100\% (21/21) patients had a 20/40 (J5) or better Bin UNVA, and at 3 months, $100 \%$ (12/12) continued to have a Bin UNVA oculus uterque of 20/40 or better. At 6 months, $75 \%(9 / 12)$ had a Bin UNVA of at least 20/40 (J5), with 50\% $(6 / 12)$ seeing 20/25 (J2) or better.

The preoperative and postoperative values for combined MRSE are shown in Figure 3. Preoperatively, the overall MRSE was $0.1 \mathrm{D}( \pm 1.0 \mathrm{SD}$, range: -3.5 to 1.4$)$ among 21 patients. At 1 month postoperatively, the MRSE for 21 patients was $-1.3 \mathrm{D}( \pm 0.8 \mathrm{SD}$, range: -2.9 to 0.6$)$ with a mean change of $-1.4 \mathrm{D}( \pm 1.6 \mathrm{SD}$, range: -4.3 to 3.5$)$ compared to preoperative data. For the eleven patients who completed 3-month postoperative visits, the MRSE was $-0.98 \mathrm{D}$ ( $\pm 0.5 \mathrm{SD}$, range: -2.1 to 0 ) with a mean change of $-0.8 \mathrm{D}$ ( $\pm 1.5 \mathrm{SD}$, range: -2.1 to 3.5 ). In addition, for the eleven patients who completed 6-month follow-up appointments, the MRSE was $-0.6 \mathrm{D}( \pm 0.4 \mathrm{SD}$, range: -1.4 to -013$)$ with a mean change of $-0.4 \mathrm{D}( \pm 1.2 \mathrm{SD}$, range: -1.5 to 2.6$)$.

\section{Efficacy}

Preoperatively, 52\% (11/21) of patients were hyperopic, $33 \%$ (7/21) were myopic, and 14\% (3/21) were emmetropic. After 1 month, 5\% (1/21) were hyperopic, 86\% (18/21) were myopic, and 10\% (3/21) were emmetropic. Of the patients who completed the 3-month follow-up, none were hyperopic,

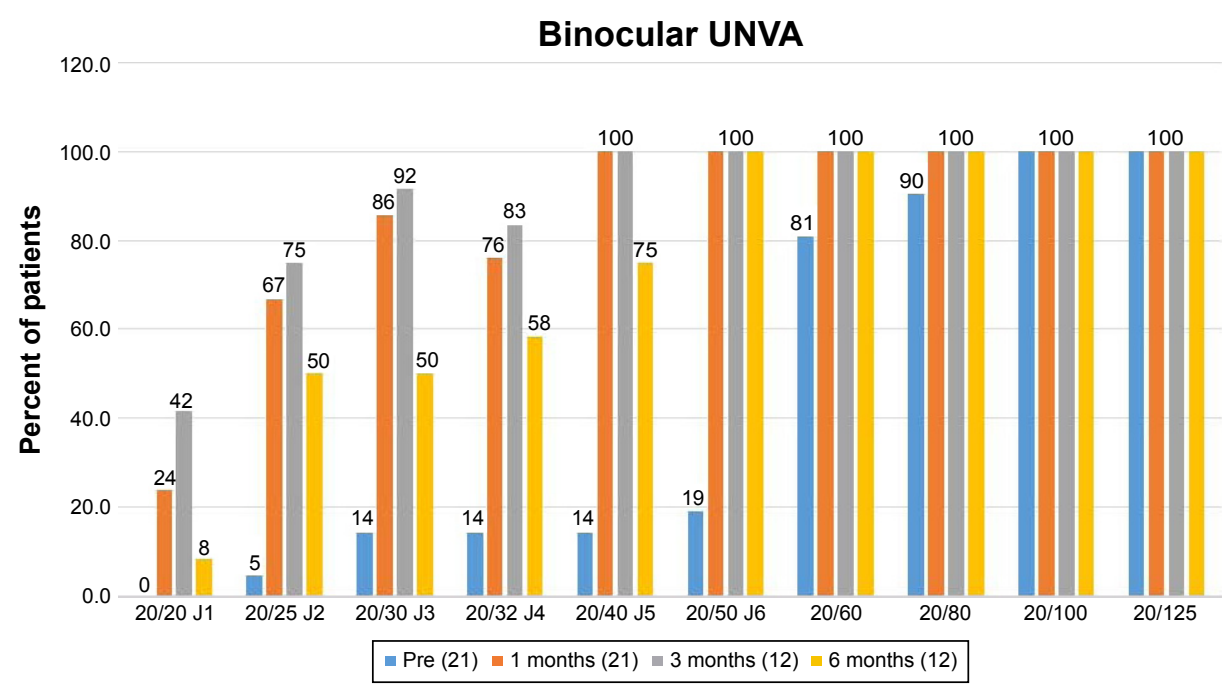

Figure 2 Cumulative binocular UNVA shown before KAMRA inlay implantation and at I, 3, and 6 months postoperatively. Abbreviation: UNVA, uncorrected near visual acuity. 


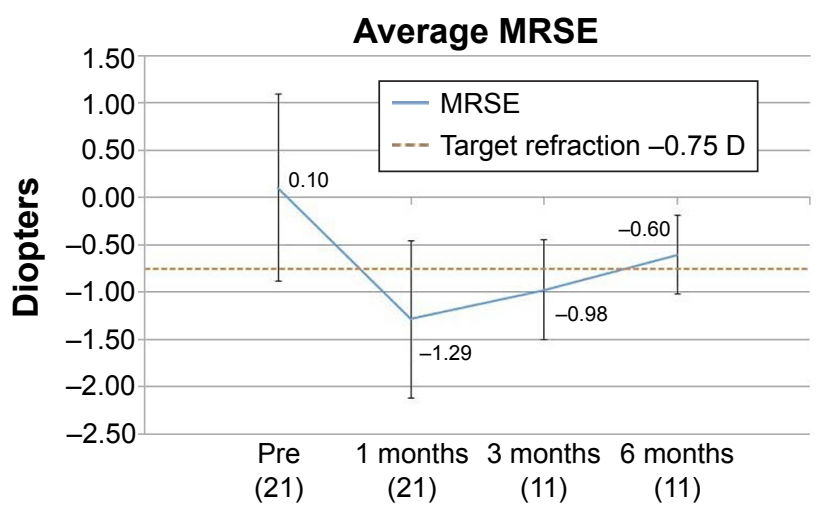

Figure 3 Cumulative preoperative MRSE and changes at I, 3, and 6 months postoperatively.

Abbreviation: MRSE, mean refractive spherical equivalent.

$91 \%(10 / 11)$ were myopic, and $9 \%(1 / 11)$ were emmetropic. By 6 months, 100\% (11/11) were myopic. Overall, 91\% (10/11) of patients were within a $\pm 0.5 \mathrm{D}$ range of our target $-0.75 \mathrm{D}$ for KAMRA use after 6 months.

At 3 months, the MRSE for the hyperopic subgroup $(\mathrm{n}=5)$ was $-0.9 \mathrm{D}( \pm 0.3 \mathrm{SD}$, range: -1.4 to -0.6$)$, the MRSE for the emmetropic subgroup $(\mathrm{n}=2)$ was $-1.6 \mathrm{D}( \pm 0.6 \mathrm{SD}$, range: -2.1 to -1.1$)$, and the MRSE for the myopic subgroup $(\mathrm{n}=4)$ was $-0.7 \mathrm{D}( \pm 0.5 \mathrm{SD}$, range: -1.1 to 0$)$. At 6 months, the MRSE was $-0.3 \mathrm{D}( \pm 0.2 \mathrm{SD}$, range: -0.1 to -0.6$)$ for the hyperopic subgroup $(\mathrm{n}=5),-1.1 \mathrm{D}( \pm 0.2 \mathrm{SD}$, range: -1.4 to -1.0$)$ for the emmetropic subgroup ( $\mathrm{n}=2)$, and $-0.7 \mathrm{D}$ ( $\pm 0.4 \mathrm{SD}$, range: -0.9 to -0.1 ) for the myopic subgroup $(n=4)$. Eighty percent $(4 / 5)$ of the hyperopic subgroup, $50 \%$ $(1 / 2)$ of the emmetropic subgroup, and $75 \%$ (3/4) of the myopic subgroup were within $\pm 0.5 \mathrm{D}$ of our $-0.75 \mathrm{D}$ target at 3 months. By 6 months, $100 \%(5 / 5)$ of the hyperopic subgroup, $50 \%(1 / 2)$ of the emmetropic subgroup, and $100 \%$ (4/4) of the myopic subgroup met the targeted range.

\section{Safety}

No patients lost two or more Snellen lines of Mono CNVA at 1, 3, or 6 months. At 1 month, 38\% (8/21) lost one line, and at 3 months, $18 \%(2 / 11)$ lost one line. At 6 months, $17 \%$ $(2 / 12)$ lost one line. We had no incidence of corneal haze, secondary steroid responders, epithelial ingrowth around the corneal pocket, recentration, or explantation among our patient groups.

\section{Discussion}

Many patients who visit ophthalmic practices seeking refractive surgery to mitigate presbyopia do not fit within the narrow range of the KAMRA inlay of -0.75 to $+0.5 \mathrm{D}$. Many of these patients have preoperative diagnosis of myopia or hyperopia with or without astigmatism. Consequently, both PRK and LASIK are able to reduce the refractive error of patients into this range to effectively treat presbyopia with the KAMRA inlay.

Despite a small sample size and a lack of 12-month data (we limited the focus to 6 months as we only had one patient with 12-month data), our results in performing PRKamra have thus far met the standards of the FDA clinical trials. The primary effectiveness criterion for the KAMRA corneal inlay, as established by the FDA clinical trials, is a Mono UNVA of 20/40 (J5) or better in $75 \%$ of eyes 12 months after surgery, of which we achieved $84 \%(n=478) .{ }^{8}$ Of our patients who had undergone simultaneous PRKamra and completed 6-month follow-up, 83\% (10/12) had a Mono UNVA of 20/40 or better and 75\% (9/12) saw Bin UNVA of $20 / 40$ or better. Although there was a slight decline from the $92 \%(11 / 12)$ of patients who had a Mono UNVA of 20/40 or better at 3 months shown in Figure 2, these 6-month results are still promising.

We have also thus far met the safety standards of the FDA clinical trials. According to their guidelines, $<5 \%$ of eyes should lose two or more lines of Mono CDVA at 12 months postoperatively, and in our results, $0.6 \%(3 / 479)$ fit this criterion. ${ }^{4}$ In our results, we found that none of our patients lost two or more Snellen lines of Mono CDVA, yet $17 \%(2 / 12)$ lost one line of sight at 6 months. However, as the epithelium and stroma postPRK continue to heal, the eyes may require more time before returning to the baseline value. As a result, we expect these results to continue to improve by the 1-year mark. In addition, based on our experiences, we have found that a deeper pocket depth can have an influence on stromal recovery and think that if we placed all our KAMRA inlays within a range of $40 \%-45 \%$ depth of the cornea thickness while still respecting the $250 \mu \mathrm{m}$ residual stromal bed, we may have better results.

The loss of vision might also be explained by a misalignment of the KAMRA compared to the purkinje reflex. However, we did not conduct a study to correlate the $\mathrm{X}$ and $\mathrm{Y}$ coordinates of the purkinje reflex with respect to the KAMRA inlay centration as it is beyond the scope of our small-group research. With further studies and more data, more effective correlations between the inlay distance from the purkinje and the loss of lines of vision may be analyzed.

Despite a small sample size, we found the most success in reducing the refractive error of the hyperopic and myopic subgroups into our targeted range. One hundred percent (5/5) of preoperatively hyperopic subgroup met the $\pm 0.5 \mathrm{D}$ range at 6 months. In addition, 100\% (4/4) of the myopic subgroup 
met the targeted range. In comparison, only $50 \%(1 / 2)$ of the emmetropic subgroup met our range.

Previous surgical methods including monovision LASIK have been used in an attempt to mitigate the effects of presbyopia. Nevertheless, monovision LASIK may result in blurred night vision and difficulty reading at intermediate distances. ${ }^{9,10}$ Despite its advantage over monovision LASIK in improving depth of field, the KAMRA inlay is only approved for a small window of patients with between -0.75 and $+0.5 \mathrm{D}$ with $<0.75 \mathrm{D}$ of cylinder. Naturally, consecutive PRK and KAMRA or LASIK and KAMRA are attractive options to bring patients into this range, while treating presbyopia with the inlay.

However, when the PRK and KAMRA procedures are performed separately, patients require 3 months of topical corticosteroids for PRK and an additional 3 months of steroids for the KAMRA procedure. Instead of using two steroid doses for 6 months after the surgeries are performed consecutively, performing these procedures simultaneously allows the patient to use one dose of steroids for 3 months. As a result, simultaneous PRKamra could help to prevent the overuse of steroids, reducing the risk of increased intraocular pressure and could be more convenient for patient recovery time than the consecutive procedures.

Understanding the appeal of shorter recovery times with LASIK compared to PRK, many surgeons first attempted to alleviate the recovery time issue through simultaneous LASIK and KAMRA. Originally, the inlay was placed under the LASIK corneal flap; however, AcuFocus now recommends that the inlay needs to be placed deeper into the cornea using a pocket. ${ }^{11}$ Thus, two depths are required, one for the normal flap for LASIK $\sim 100 \mu \mathrm{m}$ and the KAMRA inlay pocket with a minimum of $200 \mu \mathrm{m}$ needed to be effective. Nevertheless, due to these two pocket depths with simultaneous LASIK and KAMRA (LASIKamra), there may be a potential risk due to the small distance between the corneal flap and the KAMRA inlay pocket. With its comparatively shallower procedural depths, PRK surgery is an attractive option to move patients within the range for the KAMRA inlay procedure and avoid the difficulties of inlay implementation with simultaneous LASIKamra.

Compared to simultaneous LASIKamra, PRKamra can also be done on patients despite the amount of ablation that the cornea may require to correct preoperative myopia or hyperopia. When performing LASIK or KAMRA inlay procedures, it is traditionally recommended to leave a residual stromal bed in the cornea of at least $250-300 \mu \mathrm{m}$. In addition, the ideal pocket depth of a KAMRA inlay is at minimum $200 \mu \mathrm{m}$, and the LASIK flap requires $100 \mu \mathrm{m}$. Due to these depths and the minimum $250 \mu \mathrm{m}$ stromal bed, if a significant amount of ablation is required in a $540 \mu \mathrm{m}$ cornea, the remainder of the cornea anterior to the KAMRA inlay may no longer be $200 \mu \mathrm{m}$. Although this will only have an effect when correcting for a significant amount of preoperative refractive error, PRKamra avoids this issue due to its much shallower depths in the epithelium removal portion of the procedure compared to the LASIK flap.

\section{Conclusion}

Our preliminary results are very promising as they have thus far met the standards of the FDA clinical trials. PRKamra holds many advantages including the shallower removal of the epithelium compared to LASIK and the shorter period of steroid use compared to the procedures performed nonsimultaneously. The results that we have found from this study are clinically adequate, yet we believe that future long-term controlled studies of consecutive versus simultaneous PRK and KAMRA are necessary to produce better predictability.

\section{Disclosure}

This study was supported in part by an unrestricted grant from Research to Prevent Blindness, New York, NY, USA. Phillip C Hoopes Jr, MD, owns shares in AcuFocus, Inc. The remaining authors have no financial interest in the materials or methods presented in this work.

\section{References}

1. Kempen JH Mitchell P, Lee KE, et al; Eye Diseases Prevalence Research Group. The prevalence of refractive errors among adults in the United States, Western Europe, and Australia. Arch Ophthalmol. 2004; 122(4):495-505.

2. Frick KD, Joy SM, Wilson DA, Naidoo KS, Holden BA. The global burden of potential productivity loss from uncorrected presbyopia. Ophthalmology. 2015;122(8):1706-1710.

3. McDonnell PJ, Lee P, Spritzer K, Lindblad AS, Hays RD. Associations of presbyopia with vision-targeted health-related quality of life. Arch Ophthalmol. 2003;121(11):1577-1581.

4. Jalali S, Aus der Au W, Shaarawy T. AcuFocus corneal inlay to correct presbyopia using Femto-LASIK. One year results of a prospective cohort study. Klin Monbl Augenheilkd. 2016;233(4):360-364.

5. Dexl AK, Jell G, Strohmaier C, et al. Long-term outcomes after monocular corneal inlay implantation for the surgical compensation of presbyopia. J Cataract Refract Surg. 2015;41(3):566-575.

6. Seyeddain O, Bachernegg A, Riha W, et al. Femtosecond laser-assisted small-aperture corneal inlay implantation for corneal compensation of presbyopia: two-year follow-up. J Cataract Refract Surg. 2013;39(2): 234-241.

7. Tomita M, Kanamori T, Waring GO 4th, Nakamura T, Yukawa S. Smallaperture corneal inlay implantation to treat presbyopia after laser in situ keratomileusis. J Cataract Refract Surg. 2013;39(6):898-905.

8. AcuFocus, Inc. KAMRA ${ }^{\circledR}$ inlay professional use information. Irvine, CA: AcuFocus, Inc. Available from: http://www.accessdata.fda.gov/ cdrh_docs/pdf12/P120023d.pdf 
9. Wright KW, Guemes A, Kapadia MS, Wilson SE. Binocular function and patient satisfaction after monovision induced by myopic photorefractive keratectomy. J Cataract Refract Surg. 1999;25(2):177-182.

10. Levinger E, Geyer O, Baltinsky Y, Levinger S. [Binocular function and patient satisfaction after monovision induced by laser in situ keratomileusis (lasik)]. Harefuah. 2006;145(3):186-190, 246-247. Hebrew.
11. Igras E, O'Caoimh R, O'Brien P, Power W. Long-term results of combined LASIK and monocular small-aperture corneal inlay implantation. J Refract Surg. 2016;32(6):379-384.
Clinical Ophthalmology

\section{Publish your work in this journal}

Clinical Ophthalmology is an international, peer-reviewed journal covering all subspecialties within ophthalmology. Key topics include: Optometry; Visual science; Pharmacology and drug therapy in eye diseases; Basic Sciences; Primary and Secondary eye care; Patient Safety and Quality of Care Improvements. This journal is indexed on

\section{Dovepress}

PubMed Central and CAS, and is the official journal of The Society of Clinical Ophthalmology (SCO). The manuscript management system is completely online and includes a very quick and fair peer-review system, which is all easy to use. Visit http://www.dovepress.com/ testimonials.php to read real quotes from published authors. 